\title{
Resistencia inducible a clindamicina en Staphylococcus aureus resistentes a meticilina aislados de pacientes pediátricos en Paraguay
}

\author{
Marlene Silvagni', Rosa Guillén², Fátima Rodríguez², Carmen Espínola', Lorena Grau' y Gladys Velázquez
}

\section{Inducible resistance to clindamycin in methicillin-resistant Staphylococcus aureus isolated from Paraguayan children}

Background: The double disc diffusion method is an alternative diagnostic that allows the identification of Staphylococcus aureus isolates apparently susceptible to clindamycin but that may develop resistance due to an induction phenomena, mainly asociated to the increase in resistance to methicillin, thus increasing the possibility of failure in the treatment. Aim: To determine the frequency of induced clindamycin resistance in methicillin-resistant S. aureus (MRSA) isolated from Paraguayan children. Materials and Methods: In this cross sectional study, we collected $145 \mathrm{~S}$. aureus isolates that caused skin and soft tissue and osteoarticular infections in pediatric patients of the Central Hospital I.P.S. in the period from December-2012 to November-2013. Resistance to clindamycin was determined by automated methods and double disc diffusion. PCR was performed for ermA, ermB, erm C and $m s r A$ genes from representative isolates. Results: The global resistance to methicillin and clindamycin was 67 and $13 \%$, respectively ( $11 \%$ attributable to the inducible mechanism). The $e r m C$ and $m s r A$ genes were detected individually in 25 and $17 \%$ of the isolates respectively while an isolate presented both genes simultaneously. Discussion: The frequency of inducible resistance to clindamycin indicates the importance of double disc diffusion methods in microbiological practice, as well as being within the cut off points considered acceptable for the use of this antibiotic for skin infections. and osteoarticular caused by MRSA.

Keywords: Methicillin-resistant Staphylococcus aureus; double disk diffusion test; susceptibility to clindamycin; pediatrics; community acquired infection.

Palabras clave: Staphylococcus aureus resistente a meticilina; método de difusión de doble disco; susceptibilidad a clindamicina; pediatría; infección comunitaria.

\section{Introducción}

S taphylococcus aureus, cocácea grampositiva, anaerobia facultativa, ampliamente distribuida por todo el mundo, se estima que coloniza a un tercio de la población global. La diversidad de factores de virulencia que posee, así como su elevada capacidad de adquirir resistencia a los antimicrobianos, exigen la necesidad de combinar métodos bioquímicos y moleculares para su estudio ${ }^{1}$.

La aparición de aislados $S$. aureus resistente a meticilina (SARM) se remonta a la década del 60 y las infecciones causadas por los mismos han aumentado en forma significativa a nivel mundial en los últimos años, constituyéndose un serio problema de salud pública ${ }^{2}$. La resistencia a meticilina es resultado de la producción de una proteína de unión a penicilina alterada (PBP2a), codificada por el gen $m e c A$, asociada a una afinidad disminuida por los $\beta$-lactámicos, lo que confiere a la bacteria resistencia a las penicilinas semisintéticas, además de todos los $\beta$-lactámicos, incluyendo cefalosporinas de primera a cuarta generación y a los carbapenemes ${ }^{3}$.

El complejo $\mathrm{MLS}_{\mathrm{B}}$, que incluye macrólidos (eritromicina), lincosamidas (clindamicina) y estreptograminas del tipo B (linezolid), es un grupo importante de antimicrobianos comúnmente empleado para el tratamiento de las infecciones por SARM. Su mecanismo de acción consiste en la inhibición de la síntesis proteica mediante una metilasa ribosomal que se une al sitio $\mathrm{P}$ en la subunidad $50 \mathrm{~S}$ del ribosoma bacteriano ${ }^{4,5}$.

La resistencia de $S$. aureus al complejo $\mathrm{MLS}_{\mathrm{B}}$ puede deberse a varios mecanismos, siendo los más importantes la expulsión activa (fenotipo $\mathrm{MS}_{\mathrm{B}}$, codificado por el gen $m s r A$ ) y la modificación del sitio activo ribosomal por acción de una metilasa (fenotipo $\mathrm{MLS}_{\mathrm{B}}$, codificado usualmente por los genes ermA o erm C), siendo este último el más común y que confiere resistencia cruzada a los tres grupos de antimicrobianos (resistencia $\left.\mathrm{MLS}_{\mathrm{B}}\right)^{5,6}$.

La expresión fenotípica de la resistencia al grupo $\mathrm{MLS}_{\mathrm{B}}$ puede ser de carácter constitutivo $\left(\mathrm{cMLS}_{\mathrm{B}}\right)$ o
'Hospital Central-Instituto de Previsión Social. Laboratorio de Microbiología y Servicio de Pediatría.

${ }^{2}$ Instituto de Investigaciones en Ciencias de la Salud, UNA. Departamento de Análisis Clínicos y Microbiología. Departamento de Biología Molecular y Biotecnología.

Los autores declaran no tener conflictos de interés Fuente de financiamiento: Fondos propios de los investigadores.

Recibido: Versión original: 6 de marzo de 2018; Segunda versión: 19 de febrero de 2019 Aceptado: 28 de mayo de 2019

\section{Correspondencia a:} Marlene Silvagni Díaz mar.sil@live.com 
inducible ( $\mathrm{iMLS}_{\mathrm{B}}$ ); ambos están relacionados con la expresión de los genes erm (erithromycin ribosome methylation $)^{4,5}$. La variable constitutiva presenta elevado grado de resistencia a cualquier antimicrobiano del grupo $\mathrm{MLS}_{\mathrm{B}}$, a diferencia de la resistencia inducida que presenta únicamente resistencia a macrólidos de 14 (eritromicina) y 15 (azitromicina) átomos y sensibilidad in vitro a macrólidos de 16 átomos, lincosamidas (clindamicina) y estreptograminas $\mathrm{B}^{4}$.

En los aislados iMLS $_{\mathrm{B}}$, la expresión del gen erm es inducida por algunos compuestos, como eritromicina, un potente inductor para la resistencia $\operatorname{iMLS}_{\mathrm{B}}$, mientras que clindamicina es un inductor débil que actúa lentamente ${ }^{7}$ Los aislados con resistencia MLS $_{B}$ aparentan susceptibilidad in vitro a clindamicina, pero al emplearse clínicamente el fármaco, ocurre in vivo la inducción de la resistencia con el consiguiente fracaso terapéutico. Esto ocurre debido a que clindamicina, como inductor débil, provoca resistencia a largo plazo. Por otro lado, eritromicina, al ser un potente inductor de resistencia, permite su utilización en pruebas de detección de aislados $\mathrm{iMLS}_{\mathrm{B}}{ }^{2}$.

Los métodos más conocidos para la identificación de resistencia al complejo $\mathrm{MLS}_{\mathrm{B}}$ son la difusión de doble disco y las pruebas moleculares como la reacción de polimerasa en cadena (RPC) para detección de los genes asociados (ermA, ermB, erm C y $m s r A)$. El método de difusión de doble disco, identifica la resistencia inducible, pudiendo presagiar la mutación hacia una resistencia constitutiva in vivo. Este tipo de resistencia no puede ser detectada usando los métodos convencionales de disco de difusión, ni por métodos de dilución en caldo o en placa convencionales $^{8,9}$. El mismo es el recomendado para la identificación de la resistencia al complejo $\mathrm{MLS}_{\mathrm{B}}$ por el Clinical and Laboratory Standards Institute (CLSI) ${ }^{10}$.

El aumento de la frecuencia de infecciones causadas por $S$. aureus resistente a meticilina (SARM) en niños atendidos en el Hospital Central del Instituto de Previsión Social (HC-IPS) ha conllevado a un incremento potencial en el uso empírico de clindamicina. En este contexto, este trabajo tuvo por objetivo determinar la frecuencia de resistencia a clindamicina inducida por eritromicina en $S$. aureus aislados de muestras clínicas de pacientes pediátricos del HC-IPS.

\section{Materiales y Métodos}

Trabajo observacional, descriptivo, de corte transversal, que incluyó a pacientes bajo 15 años de edad, internados en el Servicio de Pediatría del HC-IPS, con diagnóstico de infecciones de piel y tejidos blandos y osteo-articulares causadas por $S$. aureus, en el período comprendido entre diciembre de 2012 y noviembre de 2013. Fueron analizados los datos demográficos y clínicos de las fichas médicas de pacientes con lesiones infectadas por $S$. aureus, obtenidas en el Servicio de Microbiología del HC-IPS.

\section{Estudios de susceptibilidad a antimicrobianos y determinación de resistencia inducible a clindamicina}

Los aislados obtenidos fueron sometidos a pruebas de identificación y sensibilidad in vitro a antimicrobianos: oxacilina, vancomicina, clindamicina, eritromicina, cotrimoxazol, ciprofloxacina y rifampicina, más resistencia inducible a clindamicina por métodos automatizados, empleando el equipo VITEK II compact (Biomeriux, Francia). A los aislados de $S$. aureus seleccionados se les realizó el método de difusión de doble disco estandarizado según pautas CLSI $^{10,11}$. En una placa de agar MüellerHinton previamente inoculada con una suspensión $(0,5$ Mc Farland) de $S$. aureus, se colocó un disco de eritromicina $(15 \mu \mathrm{g})$ y otro de clindamicina $(2 \mu \mathrm{g})$ separados por una distancia de $15 \mathrm{~mm}$ de borde a borde. Después de $24 \mathrm{~h}$ de incubación a $35^{\circ} \mathrm{C}$, la presencia de un halo en forma de letra $\mathrm{D}$ en la zona del disco de clindamicina, próxima al de eritromicina (efecto zona $\mathrm{D}$ ), se consideró como fenotipo de resistencia inducible a clindamicina.

\section{Identificación de genes erm y msrA}

La detección de genes de resistencia al complejo $\mathrm{MLS}_{\mathrm{B}}$ se realizó por RPC en su formato convencional en todas las cepas de $S$. aureus resistentes a eritromicina por el método de difusión de doble disco, para lo cual los aislados fueron remitidos al Instituto de Investigaciones en Ciencias de la Salud (IICS). Se estudiaron los genes codificantes de metilasas ermA, ermB, erm $C$ y el gen responsable de un sistema de expulsión activa $m s r A$. Se realizó extracción de ADN de los aislados por el método de ebullición, siguiendo el protocolo descrito previamente por Zhang y cols., con algunas modificaciones ${ }^{12}$. El sobrenadante conteniendo el ADN fue conservado a $-20^{\circ} \mathrm{C}$.

La RPC para detección de los genes de resistencia se realizó por medio de reacciones individuales utilizando oligonucleótidos específicos para cada uno de ellos (ermA, ermB, ermC y $m s r A$ ), descritos por Lina y cols., en el año $1999^{13}$.

Las RPCs fueron realizadas bajo las siguientes condiciones: $1 \mathrm{X}$ buffer- $\mathrm{KCl}$ (Fermentas, E.U.A.), 0,5 mM dNTPs (Fermentas, E.U.A.), $2 \mu \mathrm{M}$ oligonucleótidos (Macrogen, Corea), $2 \mathrm{mM} \mathrm{MgCl}$ (Fermentas, E.U.A.), $1 \mathrm{U}$ de Taq polimerasa (Fermentas, E.U.A.) y 1,5 $\mu \mathrm{L}$ de ADN molde obtenido por el método de ebullición, con un volumen total de reacción de $25 \mu \mathrm{L}$. Las condiciones de ciclado fueron las siguientes: desnaturalización inicial a $94{ }^{\circ} \mathrm{C}$ por $5 \mathrm{~min}, 30$ ciclos de desnaturalización $\left(94{ }^{\circ} \mathrm{C}\right.$ por $45 \mathrm{seg})$, anillamiento $\left(50^{\circ} \mathrm{C}\right.$ por $\left.45 \mathrm{seg}\right)$ y extensión $\left(72{ }^{\circ} \mathrm{C}\right.$ por $\left.1 \mathrm{~min}\right)$ y una extensión final a $72{ }^{\circ} \mathrm{C}$ por 2 
min. Para ello se utilizó el termociclador Esco Healthcare (Swift Max Pro, Singapur).

Se incluyeron controles negativos para cada tanda de RPC realizadas. Sin embargo, como no se contaba con cepas de referencia portadoras de los genes citados, se procedió a realizar el siguiente abordaje: someter a la totalidad de aislados al análisis de amplificación por RPC de los cuatro genes en las condiciones descritas en la publicación fuente y aquellos que generaron productos de RPC con el tamaño esperado, fueron sometidos a secuenciación (Macrogen, Corea) y alineamiento con secuencias del Genebank empleando la herramienta BLAST (NCBI, E.U.A.), para identificación exacta del gen y posterior empleo de dicho aislado como control positivo validado para posteriores reacciones de RPC.

Para la visualización de los productos obtenidos, se procedió a realizar electroforesis en geles de agarosa al $2 \%$ en cubas electroforéticas (MUPID-3, Japón) a 110V durante $30 \mathrm{~min}$; y posterior tinción con bromuro de etidio $0,5 \mu \mathrm{g} / \mathrm{mL}^{12}$. Para la comparación de tamaños de los productos obtenidos se utilizó marcador de tamaño molecular de $50 \mathrm{pb}$ (Embiotec, Argentina). Los resultados fueron registrados en formato de fotos digitales y analizados con el software de KODAK Digital Science DC 120 (Kodak, EEUU).

\section{Aspectos éticos}

Fueron respetados los criterios éticos de confidencialidad de los pacientes ya que las muestras fueron manejadas de manera estricta bajo códigos para su identificación y no se divulgaron datos personales en forma individual. El protocolo del presente estudio contó con la aprobación del Comité de Ética en Investigación del Instituto de Previsión Social.

\section{Resultados}

Fueron incluidas 145 muestras de pacientes pediátricos; 95 (66\%) correspondían al sexo masculino, y las 50 restantes (34\%) al sexo femenino. Edad promedio de 8 años, incluyendo pacientes de un rango desde recién nacidos hasta 15 años.

La mayoría de las muestras provenía de infecciones de piel y tejidos blandos $125 / 145(86 \%)$ y en menor proporción de lesiones osteo-articulares 20/145 (14\%).

Se observó una evolución favorable en 129 pacientes, mientras que se registraron tres óbitos por cuadros sépticos, no contándose con datos clínicos completos sobre la evolución en los restantes 13 pacientes.

Los antecedentes de prescripción de antimicrobianos en esquema empírico estuvieron disponibles en 129 casos; los antibacterianos más frecuentemente empleados fueron clindamicina en $60 \%$ de los casos $(n=78)$, seguido de cefazolina o cefalexina en $29 \%(n=37)$ y amoxicilina/ ácido clavulánico en $7 \%(n=9)$. El uso de vancomicina se registró en tres niños $(2 \%)$, mientras que antimicrobianos como meropenem y cotrimoxazol fueron empleados con frecuencia muy espóradica, menor a $1 \%$.

De los 145 aislados de $S$. aureus analizados, 97 (67\%) fueron identificados como SARM y 19 (13\%) como resistentes a clindamicina. En referencia a los mecanismos de resistencia a clindamicina el análisis fenotípico mostró datos compatibles con resistencia inducible por eritromicina ( iMLS $_{\mathrm{B}}$ ) en $11 \%$ de los aislados $(16 / 145)$ y constitutiva $\left(\mathrm{cMLS}_{\mathrm{B}}\right)$ en $2 \%(3 / 145)$. En el caso de los aislados que presentaron el fenotipo iMLS $\mathrm{B}_{\mathrm{B}}, 13 / 16$ (81\%) correspondían a SARM y 3/16 (19\%) a $S$. aureus sensibles a meticilina (SASM). Se registró $100 \%$ de concordancia entre el sistema de alertas sobre posibles mecanismos de resistencia a clindamicina generados por el VITEK y el método de difusión de doble disco. La resistencia a otros antimicrobianos ensayados por método automatizado se muestra en la Tabla 1.

En 14 de los 16 pacientes infectados con aislados de fenotipo de resistencia iMLS ${ }_{B}$ se constató la prescripción de algún antimicrobiano en respuesta al esquema empírico, siendo el uso de clindamicina sola o en combinación con cefotaxima registrado en 9 casos.

De los aislados que presentaron resistencia $\mathrm{iMLS}_{\mathrm{B}}$, 12 permanecieron viables y fueron sometidos a análisis molecular, para detección de los siguientes cuatro genes de resistencia a macrólidos: ermA, ermB, erm C y msrA (Figura 1). Se logró la identificación de genes asociados a resistencia a clindamicina en 6 aislados $(50 \%$ del total de aislados analizados). Se detectó la presencia de los genes: $\mathrm{erm} C$ y $m s r A$ de forma individual en tres $(25 \%)$ $\mathrm{y} \operatorname{dos}(17 \%)$ aislados, respectivamente, mientras que un aislado presentó ambos genes de forma simultánea.

La secuenciación y alineamiento de los productos de RPC de tamaños esperados para ambos genes revelaron elevada identidad (99-100\%), empleando secuencias de

Tabla 1. Perfil de susceptibilidad a los antimicrobianos ensayados en los aislados de Staphylococcus aureus analizados $(n=145)$

\begin{tabular}{lcc|}
\hline Antimicrobiano & $\begin{array}{c}\text { Resistente } \\
\mathbf{n}(\%)\end{array}$ & $\begin{array}{c}\text { Sensible } \\
\mathbf{n}(\%)\end{array}$ \\
\hline Oxacilina & $97(67 \%)$ & $48(33 \%)$ \\
Clindamicina & $19(13 \%)$ & $126(87 \%)$ \\
\hline Eritromicina & $22(15 \%)$ & $123(85 \%)$ \\
\hline Cotrimoxazol & $1(0,6 \%)$ & $144(99 \%)$ \\
Vancomicina & - & $145(100 \%)$ \\
Ciprofloxacina & - & $145(100 \%)$ \\
Rifampicina & - & $145(100 \%)$ \\
\hline
\end{tabular}




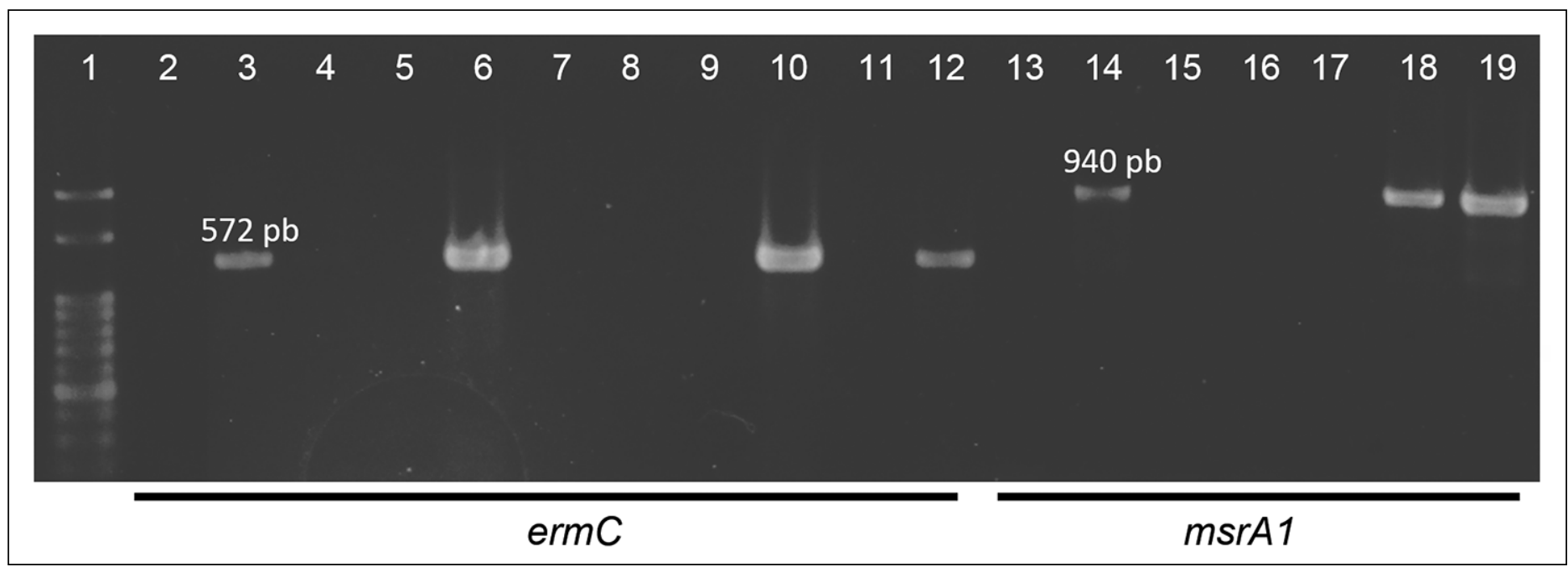

Figura 1. Productos de reacción de polimerasa en cadena con oligonucleótidos específicos para los genes ermC (572 pb) y msrA (940 pb). Carriles: 1 Marcador de tamaño molecular de 50 bp; 2 y 13: Controles negativos para cada gen; 3 y 14: Controles positivos para cada gen; 6, 10, 12: Muestras positivas para gen ermC (572 pb); 18 y 19: Muestras positivas para el gen $m s r A(940 \mathrm{pb}) ; 4,5,7,8,9,11,15,16$ y 17: Muestras negativas.

referencia del Genebank, cuyos números de acceso son: KY788638.1 para ermC y NG048001.1 para msrA. Si bien se intentó amplificar los genes ermA, ermB, no se logró empleando las condiciones descritas por los autores $\mathrm{y}$, por tanto, al no contar con aislados control positivo, no se pudieron estandarizar condiciones óptimas de amplificación para estos genes.

\section{Discusión}

La elevada frecuencia de infecciones por SARM registrada en este estudio y que se acerca a $70 \%$ de todas las infecciones por $S$. aureus, ha forzado a la incorporación cada vez más frecuente de clindamicina y otros antimicrobianos como parte de los esquemas de antibioterapia ante casos de infecciones compatibles con aquellas causadas por $S$. aureus. El uso empírico de clindamicina se dio en cerca de $60 \%$ de los pacientes incluidos en este trabajo. Estos resultados son superiores a lo reportado en dos estudios realizados en Paraguay, en el año 2010 y en el período 2012 a 2014, que refieren porcentajes de SARM de entre 19 y $54 \%$ y resistencia a clindamicina de 4 a $6 \%$, respectivamente ${ }^{14,15}$.

Del 13\% total de S. aureus aislados resistentes a clindamicina, el mecanismo inducible de resistencia es el responsable de prácticamente 9 de cada 10 casos. Frente a este hecho, es fundamental tener presente que el uso de sistemas automatizados en el laboratorio de microbiología que hace frente de forma exitosa a un elevado número de muestras a analizar, requiere del uso combinado de técnicas de microbiología convencional como el método de difusión de doble disco para la detección de ciertos mecanismos de resistencia como es el caso de iMLS $_{B}$. Las alertas brindadas por los equipos automatizados sobre la posible presencia del mecanismo iMLS $\mathrm{B}_{\mathrm{B}}$ tuvieron una excelente concordancia con el método de difusión de doble disco.

La prevalencia de este tipo de resistencia es variable según área geográfica, siendo nuestros resultados similares a lo reportado correspondiente a la resistencia iMLS $_{B}$ en diversos estudios realizados en India y Estados Unidos de América, alrededor de 9,5 al 13,1\% ${ }^{16-18}$ pero inferior a los datos de un estudio hecho en Australia donde se alcanzaron cifras de resistencia de $96,3 \%^{19-21}$.

En el análisis genotípico se encontraron los genes erm $C$, codificante de metilasas y $m s r A$, responsable de un sistema de expulsión activa, implicados en la resistencia a clindamicina. Ello coincide con los hallazgos de publicaciones internacionales comparadas a nivel regional, con excepción del Brasil, donde el gen predominante fue el ermA, al igual que en Venezuela ${ }^{5,22,23}$.

Si bien éste es el primer trabajo en Paraguay que busca identificar a nivel molecular los genes responsables del mecanismo de resistencia a clindamicina, debemos reconocer que presentamos la limitación de no contar con cepas de referencia portadoras de estos genes. Hemos logrado obtener y validar cepas portadoras de los genes erm $C$ y $m s r A$ con excelentes porcentajes de identidad respecto a las secuencias registradas en bases de datos internacionales, que serán de gran utilidad para estudios futuros. Es importante indicar que debemos realizar un 
cribado más amplio, que incluya un número mayor de aislados y que nos brinde la posibilidad de identificar cepas portadoras de otros genes de resistencia a clindamicina como sería el caso de ermA y ermB. Así mismo, en un futuro próximo deseamos poner a punto técnicas moleculares para la detección de los genes lnu $A$ y $\operatorname{lnuB}$, que codifican una enzima capaz de inactivar la acción de algunas lincosaminas por adenilación y que han sido referidas como causantes de fallas terapéuticas en el tratamiento con clindamicina ${ }^{24}$.

La detección de porcentajes de resistencia a clindamicina en este estudio ha superado el punto de corte de $10 \%$, considerado como tope razonable para el uso de clindamicina en el tratamiento empírico de las infecciones leves de piel y tejidos blandos, de probable etiología estafilocócica, de gravedad leve o moderada, producidas por SARM (o por SASM en pacientes alérgicos a los $\beta$-lactámicos $)^{11}$. Este hecho nos parece de gran importancia ya que puede tener un impacto directo en el esquema empírico a tener en cuenta dentro de la práctica pediátrica diaria.

\section{Conclusiones}

El mecanismo iMLS $_{\mathrm{B}}$ es responsable de la mayoría de los casos de resistencia a clindamicina en aislados de SARM que produjeron infecciones en niños durante el período de estudio. Si bien se ha detectado a erm C dentro de los aislados estudiados, es importante continuar con la puesta a punto y detección de otros genes responsables del fenotipo iMLS $_{\mathrm{B}}$. Los grados de resistencia a clindamicina son preocupantes debido a que se encuentran en la frontera de la tolerancia para considerar a este antimicrobiano dentro de los esquemas de prescripción empíricos para infecciones de piel y tejidos blandos compatibles con origen estafilocóccico.
Agradecimientos. A los miembros del Laboratorio de Microbiología del Hospital Central del Instituto de Previsión Social.

\section{Resumen}

Introducción: El método de difusión de doble disco se presenta como una alternativa diagnóstica que permite identificar aislados de Staphylococcus aureus susceptibles a clindamicina, ante el aumento de resistencia a meticilina, reduciendo así la posibilidad de fallo en el tratamiento. Objetivo: Determinar la frecuencia de resistencia a clindamicina inducida por eritromicina en $S$. aureus resistentes a meticilina (SARM) aislados de niños paraguayos. Materiales y Métodos: Estudio observacional, descriptivo, de corte transversal. Se colectaron 145 aislados $S$. aureus que causaron infecciones de piel y tejidos blandos y osteoarticulares en pacientes pediátricos del Hospital Central del Instituto de Previsión Social en el período de diciembre-2012 a noviembre-2013. La resistencia a clindamicina se determinó por métodos automatizados y de difusión de doble disco. Se realizó reacción de polimerasa en cadena para genes erm $A$, ermB, erm $C$ y $m s r A$ de aislados representativos. Resultados: La resistencia global a meticilina y clindamicina fue de 67 y $13 \%$, respectivamente $(11 \%$ atribuible al mecanismo de resistencia a clindamicina inducible). Los genes erm $C$ y $m s r A$ fueron detectados individualmente en 25 y $17 \%$ de los aislados, respectivamente, mientras que un aislado presentó ambos genes en simultáneo. Discusión: La frecuencia de mecanismo de resistencia inducible a clindamicina señala la importancia de los métodos de difusión de doble disco en la práctica microbiológica, así como se encuentran en los límites de puntos de cortes considerados como aceptables para el uso de este antimicrobiano para infecciones cutáneas y osteo-articulares causadas por SARM.

\section{Referencias bibliográficas}

1.- Cervantes-García E, García-González R, Salazar-Schettino P M. Características generales del Staphylococcus aureus. Rev Latin Patol Clin 2014; 61 (1): 28-40. https://www.medigraphic. com/pdfs/patol/pt-2014/pt141e.pdf.

2.- Montoya C I, Mira O M, Álvarez A I, Cofre G J, Cohen V J, Donoso W G, et al. Resistencia inducible a clindamicina en Staphylococcus aureus meticilino resistente. Rev Chil Pediatr. 2009; 80(1): 48-53. http://dx.doi.org/10.4067/ S0370-41062009000100006.

3.- Lakhundi S, Zhang K. Methicillinresistant Staphylococcus aureus: molecular characterization, evolution, and epidemiology. Clin Microbiol Rev 2018; 31 (4): e00020-18. doi: 10.1128/CMR.00020-18.
4.- Tamariz J, Agapito J, Horna G, Tapia E, Vicente W, Silva M, et al. Staphylococcus aureus resistente a meticilina adquirido en la comunidad aislados en tres hospitales de Lima-Perú. Rev Medica Hered 2010; 21 (1): 4-10. http://www.scielo.org.pe/pdf/rmh/v21n1/ v21n1ao1.pdf.

5.- Merino-Díaz L, de la Casa ÁC, Torres-Sánchez MJ, Aznar-Martín J. Detección de resistencia inducible a clindamicina en aislados cutáneos de Staphylococcus spp. por métodos fenotípicos y genotípicos. Enf Infecc Microbiol Clin 2007; 25 (2): 77-81. doi: 10.1157/13098567.

6.- Fiebelkorn K R, Crawford S A, McElmeel $\mathrm{M}$ L, Jorgensen J H. Practical disk diffusion method for detection of inducible clindamycin resistance in Staphylococcus aureus and coagulase-negative Staphylococci. J Clin
Microbiol 2003; 41 (10): 4740-4. doi: 10.1128/ jcm.41.10.4740-4744.2003

7.- Artiles Campelo F, Horcajada Herrera I, Álamo Antunéz I, Lafarga Capuz B, Cañas-Pedrosa A. Fenotipos y mecanismos genéticos de resistencia a macrólidos y lincosamidas en estreptococos del grupo viridans. Rev Esp Quimioter 2007; 20 (3): 317-22. http://www. seq.es/seq/0214-3429/25/1/artiles.pdf

8.- Castellano M, Perozo A, Molero M, Montero S, Primera F. Resistencia a clindamicina inducida por eritromicina en cepas de Staphylococcus aureus de origen clínico. Kasmera 2015; 43 (1). http://ve.scielo.org/pdf/km/v43n1/art04.pdf.

9.- Prieto Prieto J, Alou Cervera L. Macrólidos, lincosamidas y estreptograminas. Med Programa Form Médica Contin Acreditado. 2002; 8 (69): 3707-14. 
10.- Performance Standards for Antimicrobial Susceptibility Testing; M100-S27 [Internet]. $27^{\mathrm{a}}$ ed. Vol. 37. USA: Clinical and Laboratory Standard Institute; 2017.250 p. Disponible en: www.clsi.org.

11.- Mensa J, Gatell J, García-Sánchez J, Letang E, López-Suñé E, Marco F. Guía de Terapéutica Antimicrobiana 2013. 23․ ANTARES; 2013.

12.- Zhang K, Sparling J, Chow B L, Elsayed S, Hussain Z, Church D L, et al. New Quadriplex PCR of Staphylococcus aureus from coagulasenegative Staphylococci. J Clin Microbiol. 2004; 42(11): 4947-55. doi: 10.1128/JCM.42.11.49474955.2004.

13.- Lina G, Quaglia A, Reverdy M-E, Leclercq R, Vandenesch F, Etienne J. Distribution of genes encoding resistance to macrolides, lincosamides, and streptogramins among Staphylococci. Antimicrob Agents Chemother. 1999; 43(5): 1062-6. PMID: 10223914.

14.- Guillén R, Carpinelli L, Rodríguez F, Castro H, Quíñónez B, Campuzano A, et al. Staphylococcus aureus adquiridos en la comunidad: caracterización clínica, fenotípica y genotípica de aislados en niños paraguayos. Rev Chil Infectol. 2016; 33(6): 609-18. doi: 10.4067/S0716-10182016000600002.

15.- Abente S, Carpinelli L, Guillén R, Rodríguez F, Fariña N, Laspina F, et al. Frecuencia de Staphylococcus aureus meticilino resistente y del factor de virulencia PVL en pacientes ambulatorios con infección de piel y partes blandas de Asunción, Paraguay. Mem Inst Investig Cienc Salud 2016; 14 (2). http:// revistascientificas.una.py/index.php/RIIC/ article/view/1086.

16.- Sasirekha B, Usha M S, Amruta J A, Ankit S, Brinda N, Divya R. Incidence of constitutive and inducible clindamycin resistance among hospital-associated Staphylococcus aureus. 3 Biotech 2014; 4 (1): 85-9. doi: 10.1007/s13205013-0133-5.

17.- Prabhu K, Rao S, Rao V. Inducible clindamycin resistance in Staphylococcus aureus isolated from clinical samples. J Lab Physicians 2011; 3 (1): 25-7. doi: 10.4103/0974-2727.78558.

18.- Ciraj A M, Vinod P, Sreejith G, Rajani K. Inducible clindamycin resistance among clinical isolates of staphylococci. Indian J Pathol Microbiol 2009; 52 (1): 49. PMID: 19136780.

19.- Schreckenberger P C, Ilendo E, Ristow K L. Incidence of constitutive and inducible clindamycin resistance in Staphylococcus aureus and coagulase-negative Staphylococci in a community and a tertiary care hospital. J Clin Microbiol 2004; 42 (6): 2777-9. doi: 10.1128/ JCM.42.6.2777-2779.2004.

20.- Levin T P, Suh B, Axelrod P, Truant A L, Fekete T. Potential clindamycin resistance in clindamycin-susceptible, erythromycin-resistant
Staphylococcus aureus: report of a clinical failure. Antimicrob Agents Chemother 2005; 49 (3): 1222-4. doi: 10.1128/AAC.49.3.12221224.2005.

21.- O’Sullivan M V N, Cai Y, Kong F, Zeng X, Gilbert G L. Influence of disk separation distance on accuracy of the disk approximation test for detection of inducible clindamycin resistance in Staphylococcus spp. J Clin Microbiol 2006; 44 (11): 4072-6. doi: 10.1128/ JCM.01632-06.

22.- Coutinho V de L S, Paiva R M, Reiter K C, de-Paris F, Barth A L, Machado A B M P. Distribution of erm genes and low prevalence of inducible resistance to clindamycin among staphylococci isolates. Braz J Infect Dis 2010; 14 (6): 564-8. PMID: 21340296.

23.- Toledo L B S, Reyes E J P, Montes A P, Urdaneta E L T. Determinación de la resistencia a meticilina y eritromicina de cepas de Staphylococcus aureus aisladas en un hospital del estado Zulia. Rev Soc Venez Microbiol 2012; 32 (2): 88-94. http://www.scielo.org.ve/pdf/rsvm/ v32n2/art03.pdf.

24.- Mišić M, Čukić J, Vidanović D, Šekler M, Matić S, Vukašinović M, et al. Prevalence of genotypes that determine resistance of staphylococci to macrolides and lincosamides in Serbia. Front Public Health 2017; 5: 200. doi: 10.3389/fpubh.2017.00200. 